\title{
Analysing the Information Flow Properties of Object-Capability Patterns
}

\author{
Toby Murray and Gavin Lowe \\ Oxford University Computing Laboratory \\ Wolfson Building, Parks Road, Oxford, OX1 3QD, United Kingdom \\ \{toby.murray, gavin. lowe\}@comlab.ox.ac.uk
}

\begin{abstract}
We consider the problem of detecting covert channels within security-enforcing object-capability patterns. Traditional formalisms for reasoning about the security properties of object-capability patterns require one to be aware, a priori, of all possible mechanisms for covert information flow that might be present within a pattern, in order to detect covert channels within it. We show how the CSP process algebra, and its model-checker FDR, can be applied to overcome this limitation.
\end{abstract}

\section{Introduction}

The object-capability model [9] is a security architecture for the construction of software systems that naturally adhere to the principle of least authority [9], a refinement of Saltzer and Schroeder's principle of least privilege [19]. Several current research projects, including secure programming languages like $\mathrm{E}$ [9], Joe-E [8] and Google's Caja [10], and microkernel operating systems like the Annex Capability Kernel [5] and seL4 [1], implement the object-capability model to provide platforms for cooperation in the presence of mutual suspicion.

Security properties are enforced in object-capability systems by deploying security-enforcing abstractions, called patterns, much in the same way that a program's ordinary functional properties are implemented by ordinary programming abstractions and design patterns. It is therefore very important to be able to understand precisely the security properties that an individual object-capability pattern does, and does not, enforce.

For systems in which confidentiality is a primary concern, we are most often interested in those security properties that capture the ways in which information may flow within them. In object-capability applications that involve confidentiality, the information flow properties of security-enforcing object-capability patterns are of vital importance. In particular, it is necessary to be able to detect the existence of covert channels within object-capability patterns.

Whilst the formal analysis of object-capability patterns has received some attention [20], the previous formalisms that were employed require all effects that are to be reasoned about to be explicitly included in any model of an objectcapability pattern that is being analysed. Thus, in order for covert channels to be detected within an object-capability pattern, the mechanisms for covert 
information propagation must be explicitly modelled. This requires one who wishes to detect covert channels in a pattern to be aware, a priori, of the possible mechanisms for covert information flow within it.

In this paper, we show how the CSP process algebra [14], and its model checker FDR [4], can be applied to model object-capability patterns and detect covert channels within them, without forcing the programmer to enumerate the mechanisms by which information may covertly propagate. We adopt CSP for modelling object-capability systems, as opposed to what others might consider to be a more natural formalism such as the $\pi$-calculus, because we can use FDR to automatically check our properties via CSP's formal theory of refinement which, as will become evident, is integral to our understanding of both object-capability systems and information flow within them.

We conclude this section by briefly explaining the object-capability model and the fragment of CSP used in this paper. Further details about CSP can be found in [14]. In Section 2, we explain how object-capability systems can be modelled in CSP. In doing so, we present an example model of a Data-Diode pattern, from [9], that is designed to allow data to flow from low-sensitivity objects to high-sensitivity ones, whilst preventing data propagating in the reverse direction. In Section 3, we give a general definition for information flow security for object-capability systems modelled in CSP and argue that the information flow property Weakened RCFNDC for Compositions [12], which can be automatically tested in FDR, is an appropriate test to apply to such systems. Applying this test to our model from Section 2, we find that it does indeed contain covert channels, before showing how to refine the model to an implementation that passes the test. The analysis here considers only a small instance of the Data-Diode pattern composed with a handful of other objects. Therefore, in Section 4, we show how to generalise our results to systems of arbitrary size in which objects may create arbitrary numbers of other objects, applying the theory of data-independence [6]. Finally, we conclude and consider related work in Section 5.

Some proofs are omitted but appear in [11]. Thanks to Bill Roscoe for useful discussions about data-independence, and to the anonymous reviewers.

The Object-Capability Model The object-capability model [9] is a model of computation and security that aims to capture the semantics of many actual object-based programming languages and capability-based systems, including all of those mentioned in Section 1. An object-capability system is an instance of the model and comprises just a collection of objects, connected to each other by capabilities. An object is a protected entity comprising state and code that together define its behaviour. An object's state includes both data and the capabilities it possesses. A capability, $c$, is an unforgeable object reference that allows its holder to send messages to the object it references by invoking $c$.

In an object-capability system, the only overt means for objects to interact is by sending messages to each other. Capabilities may be passed between objects only within messages. In practice, object $o$ can pass one of its capabilities, $c$, directly to object $p$ only by invoking a capability it possesses that refers to $p$, 
including $c$ in the invocation. This implies that capabilities can be passed only between objects that are connected, perhaps via intermediate objects.

Each object may expose a number of interfaces, known as facets. A capability that refers to an object, $o$, also identifies a particular facet of $o$. This allows the object to expose different functionality to different clients by handing each of them a capability that identifies a separate facet, for example.

An object may also create others. In doing so, it must supply any resources required by the newly created object, including its code and any data and capabilities it is to possess initially. Hence, a newly created object receives its first capabilities solely from its parent. When creating an object, the parent exclusively receives a capability to the child. Thus, an object's parent has complete control over those objects the child may come to interact with in its lifetime. This is the basis upon which mandatory security policies can be enforced [9].

In object-capability operating systems like seL4, each process may be thought of as a separate object. In object-capability languages like Caja, objects are akin to those from object-oriented languages; capabilities are simply object references.

CSP A system modelled in CSP comprises a set of concurrently executing processes that execute by performing events. Processes communicate by synchronising on common events, drawn from the set $\Sigma$ of all visible events.

The process $S T O P$ represents deadlock and cannot perform any events. The process ? $a: A \rightarrow P_{a}$ is initially willing to perform all events from the set $A$ and offers its environment the choice of which should be performed. Once a particular event, $a \in A$, has been performed, it behaves like the process $P_{a}$.

CSP allows multi-part events to be defined, where a dot is used to separate each part of an event. Suppose we define the set of events $\{$ plot. $x . y \mid x, y \in \mathbb{N}\}$. Then the process plot? $x ? y \rightarrow S T O P$ offers all plot events whilst the process plot? $x:\{1, \ldots, 5\} ! 3 \rightarrow S T O P$ offers all events from $\{$ plot. $x .3 \mid x \in\{1, \ldots, 5\}\}$. $\left\{\left|c_{1} \ldots c_{k}\right|\right\}$ denotes the set of events whose first $k$ components are $c_{1}, \ldots, c_{k}$.

The process $P \square Q$ can behave like either the process $P$ or the process $Q$ and offers its environment the initial events of both processes, giving the environment the choice as to which process it behaves like. The process $P \sqcap Q$ can also behave like either $P$ or $Q$ but doesn't allow the environment to choose which; instead, it makes this choice internally. $P \backslash A$ denotes the process obtained when $P$ is run but all occurrences of the events in $A$ are hidden from its environment.

The process $P \| Q$ runs the processes $P$ and $Q$ in parallel forcing them to synchronise on all events from the set $A$. The process $S=\|_{1 \leq i \leq n}\left(P_{i}, A_{i}\right)$ is the alphabetised parallel composition of the $n$ processes $P_{1}, \ldots, \bar{P}_{n}$ on their corresponding alphabets $A_{1}, \ldots, A_{n}$. Each process $P_{i}$ may perform events only from its alphabet $A_{i}$, and each event must be synchronised on by all processes in whose alphabet it appears. $P_{1} A_{1} \|_{A_{2}} P_{2}$ is equivalent to $\|_{1<i<2}\left(P_{i}, A_{i}\right)$.

A process diverges when it performs an infinite number of internal $\tau$ events. A process terminates by performing the special termination event $\checkmark$. In this paper, we restrict our attention to processes that never diverge nor terminate. 
Given a CSP process $P$, traces $(P)$ denotes the set that contains all finite sequences of visible events (including all prefixes thereof) that it can perform. A stable-failure is a pair $(s, X)$ and denotes a process performing the finite sequence of events $s$ and then reaching a stable state in which no internal $\tau$ events can occur, at which point all events from $X$ are unavailable, i.e. $X$ can be refused. We write failures $(P)$ for the set that contains all stable-failures of the process $P$. We write divergences $(P)$ for the set of traces of $P$ after which it can diverge. For all of the processes $P$ that we consider in this paper, divergences $(P)=\{\}$. For any divergence-free process $P, \operatorname{traces}(P)=\{s \mid(s, X) \in$ failures $(P)\}$.

CSP's standard denotational semantic model is the failures-divergences model [14]. Here a process $P$ is represented by the two sets: failures $(P)$ and divergences $(P)$. One CSP process $P$ is said to failures-divergences refine another $Q$, precisely when failures $(P) \subseteq$ failures $(Q) \wedge$ divergences $(P) \subseteq$ divergences $(Q)$. In this case, we write $Q \sqsubseteq P$.

Sequences are written between angle brackets; \langle\rangle denotes the empty sequence. $s^{\wedge} t$ denotes the concatenation of sequences $s$ and $t . s \backslash H$ denotes the sequence obtained by removing all occurrence of events in the set $H$ from the sequence $s$. $s \uparrow H$ denotes the sequence obtained by removing all non- $H$ events from $s$.

\section{Modelling Object-Capability Systems in CSP}

In this section, we describe our approach to modelling object-capability systems in CSP. Note that we ignore the issue of object creation for now. This will be handled later on in Section 4.

We model an object-capability system System that comprises a set Object of objects as the alphabetised parallel composition of a set of processes $\{$ behaviour $(o) \mid o \in$ Object $\}$ on their corresponding alphabets $\{\alpha(o) \mid o \in$ Object $\}$. So System $=\|_{o \in \text { Object }}($ behaviour $(o), \alpha(o))$.

The facets of each object $o \in$ Object are denoted facets $(o)$. We restrict our attention to those well-formed systems in which facets $(o) \cap \operatorname{facets}(p) \neq\{\} \Rightarrow$ $o=p$. Recall that an individual capability refers to a particular facet of a particular object. Hence, we define the set Cap $=\bigcup\{$ facets $(o) \mid o \in$ Object $\}$ that contains all entities to which capabilities may refer.

The events that each process behaviour $(o)$ can perform represent it sending and receiving messages to and from other objects in the system. We define events of the form $f_{1} \cdot f_{2} . o p . a r g$ to denote the sending of a message from the object with facet $f_{1}$ to facet $f_{2}$ of the object with this facet, requesting it to perform operation $o p$, passing the argument arg and a reply capability $f_{1}$, which can be used later to send back a response. Here $f_{1}, f_{2} \in$ Cap. Arguments are either capabilities, data or the special value null, so $\arg \in \operatorname{Cap} \cup$ Data $\cup\{$ null $\}$, for some set Data of data. An operation op comes from the set $\{$ Call, Return $\}$. These operations model a call/response remote procedure call sequence in an object-capability operating system or a method call/return in an object-capability language.

The alphabet of each object $o \in$ Object contains just those events involving $o$. Hence, $\alpha(o)=\left\{\left|f_{1} \cdot f_{2}\right| f_{1}, f_{2} \in \operatorname{Cap} \wedge\left(f_{1} \in \operatorname{facets}(o) \vee f_{2} \in \operatorname{facets}(o)\right)\right\}$. 
We require that the process behaviour (o) representing the behaviour of each object $o \in$ Object adheres to the basic rules of the object-capability model, such as not being able to use a capability it has not legitimately acquired. We codify this by defining the most general and nondeterministic process that includes all permitted behaviours (and no more) that can be exhibited by an object $o$. Letting facets $=$ facets $(o)$ denote the set that comprises o's facets, and caps $\subseteq$ Cap and data $\subseteq$ Data denote the sets of capabilities and data that $o$ initially possesses, the most general process that includes all behaviours permitted by the objectcapability model that $o$ may perform is denoted Untrusted(facets, caps, data).

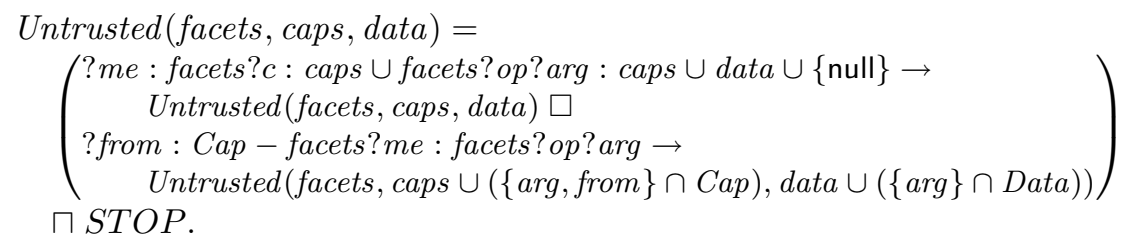

This object can invoke only those capabilities $c \in$ caps $\cup$ facets that it possesses. In doing so it requests an operation op, and may include only those arguments arg $\in$ caps $\cup$ data $\cup\{$ null $\}$ it has, along with a reply capability me $\in$ facets to one of its facets. Having done so, it returns to its previous state.

This object can also receive any invocation from any other, where the reply capability included in the invocation is from $\in$ Cap - facets, to one of its facets $m e \in$ facets, requesting an arbitrary operation op, and containing an arbitrary argument arg. If such an invocation occurs, the object may acquire the reply capability from, as well as any capability or datum arg in the argument. This process may also deadlock at any time, making it maximally nondeterministic.

The behaviour behaviour $(o)$ of an object $o \in$ Object, whose initial capabilities and data are caps(o) and data(o) respectively, is then valid if and only if all behaviours it contains are present in Untrusted(facets(o), caps(o), data(o)). This leads to the following definition of a valid object-capability system.

Definition 1 (Object-Capability System). An object-capability system is a tuple (Object, behaviour, facets, Data), where Object, behaviour, facets and Data are as discussed above and, letting Cap $=\bigcup\{$ facets $(o) \mid o \in$ Object $\}$, there exist functions caps : Object $\rightarrow \mathbf{P}$ Cap and data : Object $\rightarrow \mathbf{P}$ Data that assign minimal initial capabilities and data to each object so that, for each o $\in$ Object, Untrusted (facets(o), caps $(o)$, data(o)) $\sqsubseteq$ behaviour $(o)$.

\subsection{An Example Pattern}

We illustrate these concepts by modelling the Data-Diode pattern [9, Figure 11.2], which is designed to allow low-sensitivity objects to send data to to high-sensitivity ones whilst preventing information flowing the other way.

A data-diode is an object that has two facets, a read-facet and a writefacet $^{1}$. It stores a single datum and begins life holding some initial value. In-

\footnotetext{
${ }^{1}$ It is unclear whether the read and write interfaces should be implemented as facets of a single object or as forwarding objects of a composite object. We choose the
} 


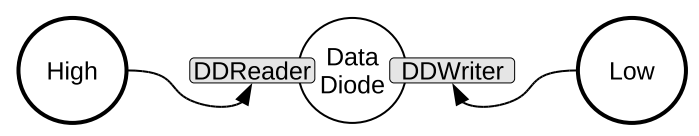

Fig. 1. A system in which to analyse the Data-Diode pattern. Bold circles indicate objects with arbitrary behaviour.

voking its read-facet causes it to return its current contents. Invoking its writefacet with an argument causes it to replace its current contents with the argument. We model a data-diode with read-facet readme and write-facet writeme that initially contains the datum val from the set Data as the CSP process ADataDiode(readme, writeme, val), defined as follows.

$$
\begin{aligned}
& \text { ADataDiode }(\text { readme, writeme, val })= \\
& \text { ?from }: \text { Cap }-\{\text { readme, writeme }\} ! \text { readme!Call!null } \rightarrow \\
& \quad \text { readme!from!Return!val } \rightarrow \text { ADataDiode }(\text { readme, writeme, val }) \\
& \text { ?from }: \text { Cap }-\{\text { readme, writeme }\} ! \text { writeme!Call? new Val }: \text { Data } \rightarrow \\
& \quad \text { writeme!from!Return!null } \rightarrow \text { ADataDiode }(\text { readme, writeme, newVal }) .
\end{aligned}
$$

Observe that this process passes Data items only, refusing all Cap arguments.

To analyse this pattern, we instantiate it in the context of the objectcapability system System depicted in Figure 1. Here, we see a data-diode, DataDiode, with read- and write-facets DDReader and DDWriter respectively. An arbitrary high-sensitivity object High has a capability to the data-diode's read-facet, allowing it to read data written by an arbitrary low-sensitivity object Low, which has a capability to the data-diode's write-facet. High and Low possess the data HighDatum and LowDatum respectively.

Let Object $=\{$ High, DataDiode, Low $\}$, HighData $=\{$ HighDatum $\}$, LowData $=\{$ LowDatum $\}$, Data $=$ HighData $\cup$ LowData, facets $($ DataDiode $)=$ $\{$ DDReader, DDWriter $\}$ and facets (other) $=\{$ other $\}$ for other $\neq$ DataDiode. The process System is then defined as explained earlier using the behaviours:

$$
\begin{aligned}
& \text { behaviour }(\text { DataDiode })=\text { ADataDiode }(\text { DDReader, DDWriter, null }), \\
& \text { behaviour }(\text { High })= \\
& \quad \text { Untrusted }(\text { facets }(\text { High }), \text { facets }(\text { High }) \cup\{\text { DDReader }\}, \text { HighData }), \\
& \text { behaviour }(\text { Low })=\text { Untrusted }(\text { facets }(\text { Low }), \text { facets }(\text { Low }) \cup\{\text { DDWriter }\}, \text { LowData }) .
\end{aligned}
$$

\section{Information Flow in Object-Capability Patterns}

Performing some basic refinement checks in FDR, which test whether certain events cannot occur in System, reveals that Low cannot obtain any HighData but that High can obtain LowDatum in this system. We now consider how to test

former option at this point and will explore the latter in Section 3.1. 
whether, despite preventing this overt flow of data, DataDiode might provide a covert channel from High to Low. We will argue that the correct property to apply to System is Weakened RCFNDC for Compositions, introduced in [12].

Information flow properties have been well-studied in the context of process algebras, including CSP (e.g. $[3,2,17,7])$. The obvious approach would take one of these properties and apply it to the process behaviour(DataDiode) to see whether it allows information to flow from its high interface DDReader to its low interface DDWriter.

However, this approach doesn't take into account the constraints imposed by the object-capability model on the objects like High and Low that may interact with DDReader and DDWriter. This is because these constraints are not reflected in behaviour(DataDiode) but are instead imposed upon behaviour (High) and behaviour (Low). For example, observe that initially the process behaviour(DataDiode) can perform the event High.DDWriter.Call.null; however, this event cannot be performed in System because it can occur there only when both High and DataDiode are willing to perform it, and behaviour (High) cannot perform it initially because High does not initially possess a capability to DDWriter. In order to get accurate results, therefore, one needs to analyse the entire system System, using an appropriate information flow property.

Recall that the processes behaviour(High) and behaviour(Low), which both instantiate the process Untrusted, are purposefully highly nondeterministic, in order to ensure that each is as general as possible. This makes the entire system System very nondeterministic. It has long been recognised that many standard information flow properties suffer from the so-called "refinement paradox" in which a property holds for a system but can be violated by one of the system's refinements. The refinements of a system capture the ways in which nondeterminism can be resolved in it. The refinement paradox is dangerous because it allows a nondeterministic system to be deemed secure when, under some resolution of the system's nondeterminism, it may actually be insecure [7].

A fail-safe way to avoid the refinement paradox is to apply an information flow property that is refinement-closed [7]. A property is refinement-closed when, for every process $P$, it holds for $P$ only if it holds for all $P$ 's refinements.

While we want to avoid the refinement paradox, refinement-closed properties are too strong for our purposes. This is because the refinements of a parallel composition include those in which the resolution of nondeterminism in one component can depend on activity that occurs within the system that the component cannot overtly observe.

For example, System is refined by a process that has the trace 〈High.High.Call.High, Low.Low.Call.null〉 but also has the stable-failure $(\langle\rangle,\{$ Low.Low.Call.null $\})$. This refinement means that System fails a number of refinement-closed information flow properties, e.g. Roscoe's Lazy Independence [14, Section 12.4] and Lowe's Refinement-Closed Failures NonDeducibility on Compositions [7]. These two behaviours arise because of the nondeterminism in Low: initially Low may either perform Low.Low.Call.null or may refuse it, depending on how this nondeterminism is resolved. In the trace 
above, where High performs the event High.High.Call.High, Low's nondeterminism is resolved such that Low.Low.Call.null occurs; while in the stable-failure, where High doesn't perform its event, this nondeterminism in Low is resolved the other way. The resolution of the nondeterminism in Low here thus depends on whether High has performed its event, in which it interacts with just itself.

A system that exhibits both of these behaviours therefore allows High's interactions with just itself to somehow influence Low. In such systems it is impossible to talk sensibly about the information flow properties of the Data-Diode pattern.

We see that in general, one cannot talk sensibly about the information flow properties of object-capability patterns without assuming that the only way for one object to directly influence another is by sending it a message or receiving one from it, since it is only overt message passing that any pattern can hope to control. Thus, in any system, we assume that the resolution of nondeterminism in any object can be influenced only by the message exchanges in which it has partaken before the nondeterminism is resolved.

Without specifying how the nondeterminism in any object may be resolved after it has engaged in some sequence $s$ of message exchanges, this therefore implies that whenever it performs $s$, the nondeterminism should be resolved consistently [12]. Two resolutions of the nondeterminism in a process after it has performed $s$ are inconsistent when it can perform some event $e$ in one but refuse $e$ in the other. Under this definition, the two different resolutions of the nondeterminism in Low above, depending on whether High has performed its event that doesn't involve Low, are inconsistent: in each case, Low performs/refuses the event Low.Low.Call.null after performing no others.

We therefore confine ourselves to the ways of resolving the nondeterminism in each object in which this kind of inconsistency does not arise. Note that these are precisely the deterministic refinements of each object, under the standard definition of determinism for CSP processes.

Definition 2 (Determinism). A divergence-free process $P$ is said to be deterministic, written $\operatorname{det}(P)$, iff $\nexists s, e \bullet s^{\wedge}\langle e\rangle \in \operatorname{traces}(P) \wedge(s,\{e\}) \in \operatorname{failures}(P)$.

With this in mind, we seek an information flow property that holds for a system System $=\|_{o \in \text { Object }}($ behaviour $(o), \alpha(o))$ just when those refinements of System, in which the nondeterminism in each object is resolved to produce a deterministic process, are deemed secure. Any such deterministic componentwise refinement may be written as System ${ }^{\prime}=\|_{o \in \text { Object }}\left(b_{o}, \alpha(o)\right)$ where $\forall o \in \operatorname{Object} \bullet$ behaviour $(o) \sqsubseteq b_{o} \wedge \operatorname{det}\left(b_{o}\right)$. Let DCRef(System) denote the set of all deterministic componentwise refinements of System. Any such refinement System ${ }^{\prime} \in$ DCRef(System) will itself be deterministic [14]. Many information flow properties, which might otherwise disagree, agree when applied to deterministic processes. Hence, given any such property Prop, we arrive at the following definition of information flow security for object-capability systems.

Definition 3. An object-capability system captured by the CSP process System $=\|_{o \in \text { Object }}($ behaviour $(o), \alpha(o))$ is secure under componen- 
twise refinement with respect to the information flow property Prop iff $\forall$ System $^{\prime} \in$ DCRef $($ System $) \bullet \operatorname{Prop}\left(\right.$ System $\left.^{\prime}\right)$.

\subsection{Testing Information Flow}

The information flow property Weakened RCFNDC for Compositions [12] is equivalent to Definition 3 when Prop is Lowe's Refinement-Closed Failures NonDeducibility on Compositions [7] (RCFNDC). RCFNDC is equivalent when applied to deterministic processes to a number of standard information flow properties, including [16] at least all those that are no stronger than Roscoe's Lazy Independence [14, Section 12.4] and no weaker than Ryan's traces formulation of noninterference [18, Equation 1]. Therefore, we adopt Weakened RCFNDC and its associated automatic refinement check [12] to test for information flow here.

Like similar information flow properties, given two sets $H$ and $L$ that partition the alphabet of a system, Weakened RCFNDC tests whether the occurrence of events from $H$ can influence the occurrence of events from $L$. In [12], it is shown that any divergence-free alphabetised parallel composition $S=\|_{1 \leq i \leq n}\left(P_{i}, A_{i}\right)$ satisfies Weakened RCFNDC, written $W R C F N D C(S)$, iff:

$$
\begin{aligned}
& \nexists s, l \bullet s \uparrow H \neq\langle\rangle \wedge l \in L \wedge \\
& \quad\left(\begin{array}{l}
s^{\wedge}\langle l\rangle \in \operatorname{traces}(S) \wedge s \backslash H \in \operatorname{traces}(S) \wedge \\
\exists i \bullet l \in A_{i} \wedge s \uparrow A_{i} \neq s \backslash H \uparrow A_{i} \wedge\left(s \backslash H \uparrow A_{i},\{l\}\right) \in \operatorname{failures}\left(P_{i}\right)
\end{array}\right) \vee \\
& \left(\begin{array}{l}
s \backslash H^{\wedge}\langle l\rangle \in \operatorname{traces}(S) \wedge s \in \operatorname{traces}(S) \wedge \\
\exists i \bullet l \in A_{i} \wedge s \uparrow A_{i} \neq s \backslash H \uparrow A_{i} \wedge\left(s \uparrow A_{i},\{l\}\right) \in \operatorname{failures}\left(P_{i}\right)
\end{array}\right) .
\end{aligned}
$$

Let $H=\left\{h\right.$.DDReader, DDReader. $h, h . h^{\prime} \mid h, h^{\prime} \in$ facets(High) $\}$ denote the set of events that represent High interacting with DDReader and itself. Similarly let $L=\left\{l l\right.$.DDWriter.Call.arg, DDWriter.l.Return.null, $l . l^{\prime} \mid l, l^{\prime} \in$ facets(Low) $\}$. Then a refinement check in FDR reveals that System from Section 2.1 can perform no events outside of $H \cup L$. This implies, for example, that neither High nor Low can obtain a capability to the other. Therefore, $H$ and $L$ partition the effective alphabet of System.

Applying the refinement check for Weakened RCFNDC to System with these definitions of $H$ and $L$, using FDR, reveals that Weakened RCFNDC doesn't hold. Interpreting the counter-example returned from FDR, we see that System can perform the trace 〈Low.DDWriter.Call.LowDatum〉 but also has the failure ( $\langle$ High.DDReader.Call.null $\rangle,\{$ Low.DDWriter.Call.LowDatum $\})$. This indicates that initially Low can invoke DDWriter but that if High invokes DDReader, it can cause Low's invocation to be refused. This occurs because DataDiode cannot service requests from High and Low at the same time. This constitutes a clear covert channel, since High can signal to Low by invoking DDReader which alters whether Low's invocation is accepted.

Low may be unable to observe this covert channel in some object-capability systems, e.g. those in which a sender of a message is undetectably blocked until the receiver is ready to receive it. For this kind of system, one might wish to replace Prop with another property, such as Focardi and Gorrieri's Traces NDC [3], 
that detects only when high events can cause low events to occur, rather than also detecting when they can prevent them from occurring as happens in the counter-example above. Modifying Weakened RCFNDC to do so simply involves removing the second disjunct from Equation 1. However, we choose to make the conservative assumption that this counter-example represents a valid fault.

Correcting the fault here involves modifying the data-diode implementation so that its interfaces for writing and reading, DDWriter and DDReader, can be used simultaneously. We do so by promoting these interfaces from being facets of a single process to existing as individual processes in their own right. These processes simply act now as proxies that forward invocations to the facets of an underlying ADataDiode process, as depicted in Figure 2.

The behaviour of a proxy me that forwards invocations it receives using the capability target is given by the process AProxy(me,target) defined as follows.

$$
\begin{aligned}
& \text { AProxy }(\text { me }, \text { target })=\text { ?from }: \text { Cap }-\{\text { me }\} ! \text { me } ! \text { Call? arg }: \text { Data } \cup\{\text { null }\} \rightarrow \\
& \quad \text { me!target!Call!arg } \rightarrow \text { target } ! \text { me } ! \text { Return? res }: \text { Data } \cup\{\text { null }\} \rightarrow \\
& \quad \text { me!from!Return!res } \rightarrow \text { AProxy }(\text { me, target }) .
\end{aligned}
$$

The data-diode is now a composite of three entities, DDReader, DDWriter and DataDiode, and as such is referred to as DDComposite. We model the system depicted in Figure 2 as an object-capability system comprising the objects from Object $=$ \{High, DDComposite, Low $\}$, where facets(DDComposite) $=\{$ DDReader, DDWriter, DDR, DDW $\}$ and, letting $R=\{\mid$ DDReader. $x, x$.DDReader $\mid x \in$ facets(DDComposite) $-\{$ DDReader $\} \mid\}$, $W=\{$ DDWriter. $x, x$.DDWriter $\mid x \in$ facets (DDComposite) $-\{$ DDWriter $\}\}$, $D D=A$ DataDiode(DDR, DDW, null) and the other definitions be as before,

$$
\begin{aligned}
& \text { behaviour }(\mathrm{DDC} \text { Composite })= \\
& \qquad\left(\left(A \operatorname{Proxy}(\mathrm{DDR} \text { eader, DDR }) \|_{R} D D\right) \|_{W} A \operatorname{Proxy}(\mathrm{DDW} \text { riter, DDW })\right) \backslash(R \cup W) .
\end{aligned}
$$

DDComposite is formed by taking the two proxies, DDReader and DDWriter, and composing them in parallel with DataDiode, whose read- and write-interfaces are now DDR and DDW respectively. Notice that we then hide the internal communications within DDComposite since these are not visible to its outside environment and it is unclear how to divide these events between the sets $H$ and $L$. FDR can be used to check that this system, System, satisfies Definition 1.

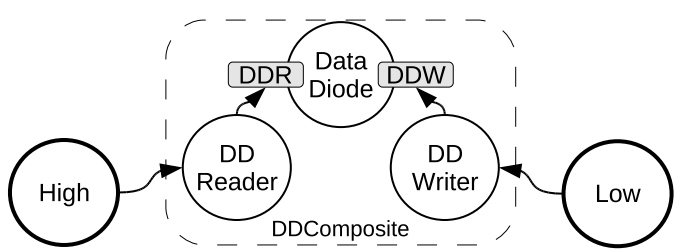

Fig. 2. An improved Data-Diode implementation. 
Performing the appropriate refinement checks in FDR reveal that High can acquire LowDatum but Low cannot acquire any HighData, and that System can perform no events outside of $H \cup L$, as before. FDR reveals that Weakened RCFNDC holds for System. Hence, we are unable to detect any covert channels in this model of the improved Data-Diode implementation.

\section{Generalising the Results}

We have verified this improved Data-Diode implementation in the context of only a handful of other objects and in the absence of object creation. In this section, we show how to generalise our analysis to all systems that have the form of Figure 3, and have arbitrary HighData and LowData. Here, the objects within each cloud can be interconnected in any way whatsoever; however, the only capability to an object outside of the high object cloud that each high object may possess is DDReader. The same is true for the low objects and DDWriter. This figure captures all systems containing an arbitrary number of high- and lowsensitivity objects and, thus, all those in which each object may create arbitrary numbers of others that share its level of sensitivity.

Roughly, the approach we take is to show that the improved system analysed in the previous section is a safe abstraction of all systems captured by Figure 3, such that if the safe abstraction is deemed secure then so will all of the systems it abstracts. For one system System ${ }^{\prime}=\|_{o \in \text { Object }^{\prime}}$ behaviour $\left.^{\prime}(o), \alpha^{\prime}(o)\right)$ to be a safe abstraction of another System $=\|_{o \in \text { Object }}($ behaviour $(o), \alpha(o))$, we require that if System' is deemed secure, then so must System.

Recall that, by Definition 3, System is secure iff $\operatorname{Prop}\left(\operatorname{System}_{D}\right)$ holds for all System ${ }_{D} \in$ DCRef(System) for some information flow property Prop. We therefore insist that in order for System ${ }^{\prime}$ to be a safe abstraction of System, that each System $_{D}$ is also present in DCRef $\left(\right.$ System $\left.^{\prime}\right)$.

Definition 4 (Safe Abstraction). Given any System ${ }^{\prime}$ and System as above, System $^{\prime}$ is a safe abstraction of System iff DCRef $($ System $) \subseteq$ DCRef $\left(\right.$ System $\left.^{\prime}\right)$.

We now show that each system System captured by Figure 3 can be safely abstracted by a system System ${ }^{\prime}$ of the form of Figure 2. We form System ${ }^{\prime}$ by taking each cloud of objects in System and aggregating all of the objects in the

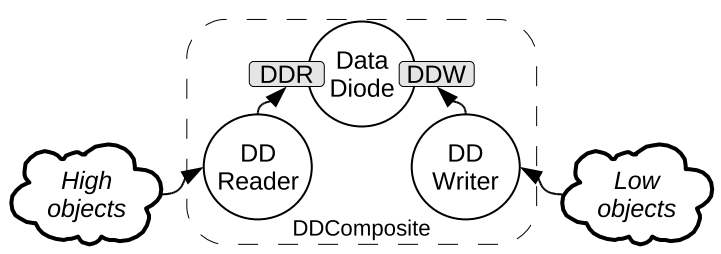

Fig. 3. Generalising the results. 
cloud into a single object in System ${ }^{\prime}$. In order to be a proper aggregation, each object from System' must have all facets, capabilities, data and behaviours of all the objects from System that it aggregates. We formally capture that System ${ }^{\prime}$ is an aggregation of System via a surjection Abs: Object $\rightarrow$ Object $^{\prime}$ that maps each object of System to the object that aggregates it in System ${ }^{\prime}$.

Definition 5 (Aggregation). Let (Object, behaviour, facets, Data) and (Object', behaviour' ${ }^{\prime}$,facets' ${ }^{\prime}$, Data) be two object-capability systems with identical sets of data, captured by System $=\|_{o \in \text { Object }}($ behaviour $(o), \alpha(o))$ and System $^{\prime}=\|_{o \in \text { Object }^{\prime}}\left(\right.$ behaviour $\left.^{\prime}(o), \alpha^{\prime}(o)\right)$ respectively. Then the second is an aggregation of the first when there exists some surjection Abs : Object $\rightarrow$ Object $^{\prime}$ such that for all $o^{\prime} \in$ Object $^{\prime}$, facets ${ }^{\prime}\left(o^{\prime}\right)=\bigcup\left\{\right.$ facets $\left.(o) \mid o \in A b s^{-1}\left(o^{\prime}\right)\right\}$ and

$$
\begin{aligned}
& \forall s \in \operatorname{traces}(\text { System }) \bullet \forall X \in \mathbf{P} \Sigma \bullet \\
& \quad\left(s \uparrow \alpha^{\prime}\left(o^{\prime}\right), X\right) \in \text { failures }\left(\|_{o \in \text { Abs }^{-1}\left(o^{\prime}\right)}(\text { behaviour }(o), \alpha(o))\right) \Rightarrow \\
& \quad\left(s \uparrow \alpha^{\prime}\left(o^{\prime}\right), X\right) \in \operatorname{failures}\left(\text { behaviour }^{\prime}\left(o^{\prime}\right)\right),
\end{aligned}
$$

where $\operatorname{Abs}^{-1}\left(o^{\prime}\right)=\left\{o \mid o \in\right.$ Object $\wedge$ Abs $\left.(o)=o^{\prime}\right\}$.

The proof of the following theorem requires some technical results beyond the scope of this paper; given limitations on space, it can be found in [11].

Theorem 1. Let System and System ${ }^{\prime}$ capture two object-capability systems as stated in Definition 5. Then if System ${ }^{\prime}$ is an aggregation of System, it is also a safe abstraction of System.

We claim that any finite collection $K \subseteq$ Object of objects $\|_{o \in K}($ behaviour $(o), \alpha(o))$, can be aggregated by a single object with behaviour Untrusted $\left(\bigcup_{o \in K}\right.$ facets $(o), \bigcup_{o \in K} \operatorname{caps}(o), \bigcup_{o \in K}$ data $\left.(o)\right)$ that has all of their capabilities, data and facets. Briefly, by Definition 1, behaviour $(o) \sqsubseteq$ Untrusted(facets(o), caps(o),data(o)) for each $o \in K$ for some sets caps(o) and $\operatorname{data}(o)$ of capabilities and data that it possesses initially. Further Untrusted $\left(\right.$ facets $(o) \cup$ facets $\left(o^{\prime}\right)$, caps $(o) \cup \operatorname{caps}\left(o^{\prime}\right)$, data $\left.(o) \cup \operatorname{data}\left(o^{\prime}\right)\right) \sqsubseteq$ Untrusted (facets $(o)$, caps $(o)$, data $(o)) \quad \alpha(o) \|_{\alpha\left(o^{\prime}\right)} \quad \operatorname{Untrusted}\left(\right.$ facets $\left(o^{\prime}\right)$, caps $\left(o^{\prime}\right)$, $\left.\operatorname{data}\left(o^{\prime}\right)\right)$. The claim then follows by induction on the size of $K$.

So consider any system that has the form of Figure 3 and let $T$ denote the facets of the high objects, $U$ the facets of the low objects and $V=$ HighData $\cup$ LowData, i.e. $V=$ Data. Then this system can be safety abstracted by a system System $_{T, U, V}$ of the form of Figure 2 in which facets $($ High $)=T$, facets $($ Low $)=U$, HighData $=V$ and LowData $=V$, so that Data $=V$. Notice that we allow High and Low to both possess all data in the safe abstraction in order to obtain maximum generality. If we can show that System $_{T, U, V}$ is secure for all non-empty choices of $T, U$ and $V$, by Theorem 1 , we can conclude that the improved Data-Diode implementation is secure in all systems captured by Figure 3 with arbitrary HighData and LowData. 
The theory of data-independence [6] can be applied to show that a property Prop holds of a process $P_{T}$, parameterised by some set $T$, for all non-empty choices of $T$, if $\operatorname{Prop}\left(P_{T}\right)$ can be shown for all non-empty $T$ of size $N$ or less, for some $N . N$ is called the data-independence threshold for $T$ for $\operatorname{Prop}\left(P_{T}\right)$. The theory requires that $P_{T}$ be data-independent in $T$, meaning roughly that $P_{T}$ handles members of the type $T$ uniformly, not distinguishing one particular value of $T$ from another. We apply data-independence theory to show that thresholds of size 1, 2 and 2 for $T, U$ and $V$ respectively are sufficient to demonstrate the security of System ${ }_{T, U, V}$ for all non-empty choices of each set.

We will use the following standard result. Let $P_{T}$ be a process that is dataindependent in some set $T$ and satisfies NoEqT for $T$, meaning that it never needs to test two values of $T$ for equality. Let $\phi$ be a surjection whose domain is $T$, where we write $\phi(T)$ for $\{\phi(t) \mid t \in T\}$ and $\phi^{-1}(X)$ for $\{y \mid y \in T \wedge \phi(y) \in X\}$. Then [6, Theorem 4.2.2], lifting $\phi$ to events and traces,

$$
\left\{(\phi(s), X) \mid\left(s, \phi^{-1}(X)\right) \in \operatorname{failures}\left(P_{T}\right)\right\} \subseteq \operatorname{failures}\left(P_{\phi(T)}\right) .
$$

Theorem 2. Let $S_{T}=\|_{1<i<n}\left(P_{T, i}, A_{T, i}\right)$ be an alphabetised parallel composition, whose components and alphabets are polymorphically parameterised by some set $T$, such that $S_{T}$ and each $P_{T, i}$ are data-independent in $T$ and satisfy NoEqT for $T$. Also let $H_{T}$ and $L_{T}$ be two sets polymorphically parameterised by $T$ that partition the alphabet of $S_{T}$ for all non-empty $T$. Let $W$ denote the maximum number of distinct elements of $T$ that appear in any single event from $L_{T}$. Then $W+1$ is a sufficient data-independence threshold for $T$ for $\operatorname{WRCFNDC}\left(S_{T}\right)$.

Proof. Assume the conditions of the theorem. Suppose for some $T$ with size greater than $W, S_{T}$ fails Weakened RCFNDC for $H_{T}$ and $L_{T}$. Then let $\tilde{T}=\left\{\tilde{t}_{0}, \ldots, \tilde{t}_{W}\right\}$ for fresh elements $\tilde{t}_{0}, \ldots, \tilde{t}_{W}$. We show that $S_{\tilde{T}}$ fails Weakened RCFNDC for $H_{\tilde{T}}$ and $L_{\tilde{T}}$.

Let $\phi: T \rightarrow \tilde{T}$ be a surjection; we fix the choice of $\phi$ below. Lift $\phi$ to events by applying $\phi$ to all components of type $T$. Then $\phi$ maps an event in the alphabet of $S_{T}$ to an event in the alphabet of $S_{\tilde{T}}$. Also, lifting $\phi$ to sets of events, $\phi\left(A_{T, i}\right)=A_{\tilde{T}, i}$ for $1 \leq i \leq n, \phi\left(H_{T}\right)=H_{\tilde{T}}$ and $\phi\left(L_{T}\right)=L_{\tilde{T}}$.

Observe that $S_{\phi(T)}=S_{\tilde{T}}$. So, by Equation 2, the presence of certain behaviours in $S_{T}$ implies the presence of related behaviours in $S_{\tilde{T}}$. Recall the characterisation of Weakened RCFNDC from Equation 1. Suppose $S_{T}$ fails the first disjunct of Equation 1 for $H_{T}$ and $L_{T}$. We show that $S_{\tilde{T}}$ fails this disjunct for $H_{\tilde{T}}$ and $L_{\tilde{T}}$. The second disjunct is handled similarly. Then there exists some $s, l$ and $i \in\{1, \ldots, n\}$ such that

$$
\begin{aligned}
& s \uparrow H_{T} \neq\langle\rangle \wedge l \in L_{T} \wedge s^{\wedge}\langle l\rangle \in \operatorname{traces}\left(S_{T}\right) \wedge s \backslash H_{T} \in \operatorname{traces}\left(S_{T}\right) \wedge \\
& l \in A_{T, i} \wedge s \uparrow A_{T, i} \neq s \backslash H_{T} \uparrow A_{T, i} \wedge\left(s \backslash H_{T} \uparrow A_{T, i},\{l\}\right) \in \operatorname{failures}\left(P_{T, i}\right) .
\end{aligned}
$$

Let $t_{0}, \ldots, t_{k-1}$ be the distinct members of $T$ that appear in $l$. Then $k \leq$ $W$. Choose $\phi\left(t_{i}\right)=\tilde{t}_{i}$ for $0 \leq i \leq k-1$ and let $\phi(t)=\tilde{t}_{k}$ for all other $t \in$ $T-\left\{t_{0}, \ldots, t_{k-1}\right\}$. Let $\tilde{s}=\bar{\phi}(s)$ and $\tilde{l}=\phi(l)$. Then $\tilde{s} \uparrow \tilde{H} \neq\langle\rangle \wedge \tilde{l} \in \tilde{L} \wedge$ $\tilde{l} \in A_{\tilde{T}, i} \wedge \tilde{s} \uparrow A_{\tilde{T}, i} \neq \tilde{s} \backslash \tilde{H} \uparrow A_{\tilde{T}, i}$. Applying Equation 2 to $S_{T}$, we have 
$\tilde{s}\langle\tilde{l}\rangle \in \operatorname{traces}\left(S_{\tilde{T}}\right) \wedge \tilde{s} \backslash \tilde{H} \in \operatorname{traces}\left(S_{\tilde{T}}\right)$. Further, $\{l\}=\phi^{-1}(\{\tilde{l}\})$ by construction. So, applying Equation 2 to $P_{T, i}$, we obtain $\left(\tilde{s} \backslash \tilde{H} \uparrow A_{\tilde{T}, i},\{\tilde{l}\}\right) \in \operatorname{failures}\left(P_{\tilde{T}, i}\right)$.

Set $H_{T, U, V}=\left\{\mid t\right.$.DDReader, DDReader.t,t.t. $\left.\left|t, t^{\prime} \in T\right|\right\}$ and $L_{T, U, V}=$ $\left\{\mid u\right.$.DDWriter.Call.d, DDWriter.u.Return.null, $u . u^{\prime} \mid u, u^{\prime} \in U, d \in$ Data $\cup\{$ null $\left.\} \mid\right\}$. Then System $_{T, U, V}$ and all of its components are data-independent in $T, U$ and $V$ and satisfy NoEqT for each.

Applying Equation 2, it is easily shown that $H_{T, U, V}$ and $L_{T, U, V}$ partition the alphabet of System ${ }_{T, U, V}$ for all non-empty choices of $T, U$ and $V$, if they do so when each of these sets has size 1. FDR confirms the latter to be true.

To verify Weakened RCFNDC, Theorem 2 suggests thresholds for $T, U$ and $V$ of 1,4 and 2 respectively. This threshold for $U$ arises from events in $L_{T, U, V}$ of the form $u . u^{\prime} . o p . u^{\prime \prime}$ for $u, u^{\prime}, u^{\prime \prime} \in U$.

In fact, in the proof of Theorem $2, l$ is necessarily an event in the alphabet of a process that can perform both $H_{T}$ and $L_{T}$ events. Hence, we can strengthen this theorem to take $W$ to be the maximum number of distinct values of type $T$ in all such events in $L_{T}$. For System ${ }_{T, U, V}$, this means that all events from $\left\{u\right.$ u. $u^{\prime} \mid$ $\left.u, u^{\prime} \in U \mid\right\}$ can be excluded when calculating the threshold for $U$, reducing it to 2 .

The most expensive of the 4 tests implied by these thresholds examines about 6 million state-pairs, taking around 4 minutes to compile and complete on a desktop PC; the others are far cheaper. All tests pass, generalising our results.

\section{Conclusion and Related Work}

We have shown how to apply CSP and FDR to automatically detect covert channels in security-enforcing object-capability patterns without forcing the programmer to specify the mechanisms by which information may propagate covertly.

Our approach couples the objects that implement a pattern with arbitrary, Untrusted, high- and low-sensitivity objects that exhibit all behaviours permitted by the object-capability model. This has the added advantage that we can compare how a pattern functions in different kinds of object-capability system, such as single-threaded versus concurrent systems, by simply refining the definition of the Untrusted process. Investigating how the information flow properties of patterns are affected by changing the context in which they are deployed is an obvious avenue for future work.

The assumption that objects affect each other only by passing messages means that our analysis cannot be applied to timed systems in which objects have access to a global clock, for instance. Extending this work to cover such systems may allow us to detect possible timing channels that may exist in them.

Spiessens' [20] is the only prior work of which we are aware that examines the security properties of object-capability patterns. The ideas of safe abstraction and aggregation defined in Section 4 were heavily inspired by similar ideas in [20]. Spiessens' formalism has the advantage of not requiring the use of dataindependence arguments to generalise analyses of small systems to large systems. On the other hand, our approach, unlike Spiessens', can detect covert channels in 
a pattern without forcing the programmer to specify the means by which information can propagate covertly. Instead, these means are captured by information flow properties that can be applied to any pattern being analysed.

The notion of aggregation is also similar to (the inverse of) van der Meyden's architectural refinement [21]. Finally, data-independence theory has been applied before to generalise analyses of small systems to larger systems, including to the analysis of cryptographic protocols [15] and intrusion detection systems [13].

\section{References}

1. D. Elkaduwe, G. Klein, and K. Elphinstone. Verified protection model of the seL4 microkernel. In Proceedings of VSTTE '08, pages 99-114. Springer, 2008.

2. R. Focardi. Comparing two information flow security properties. In Proceedings of CSFW'96, pages 116-122. IEEE Computer Society, 1996.

3. R. Focardi and R. Gorrieri. A classification of security properties for process algebras. Journal of Computer Security, 3(1):5-33, 1995.

4. Formal Systems (Europe), Limited. FDR2 User Manual, 2005.

5. D. Grove, T. Murray, C. Owen, C. North, J. Jones, M. R. Beaumont, and B. D. Hopkins. An overview of the Annex system. In Proceedings of ACSAC '07, 2007.

6. R. S. Lazić. A Semantic Study of Data Independence with Applications to Model Checking. D.Phil. thesis, Oxford University Computing Laboratory, 1999.

7. G. Lowe. On information flow and refinement-closure. In Proceedings of the Workshop on Issues in the Theory of Security (WITS '07), 2007.

8. A. M. Mettler and D. Wagner. The Joe-E language specification, version 1.0. Technical Report EECS-2008-91, University of California, Berkeley, August 2008.

9. M. S. Miller. Robust Composition: Towards a Unified Approach to Access Control and Concurrency Control. PhD thesis, Johns Hopkins University, 2006.

10. M. S. Miller, M. Samuel, B. Laurie, I. Awad, and M. Stay. Caja: Safe active content in sanitized JavaScript (draft), 2008.

11. T. Murray. Analysing the Security Properties of Object-Capability Patterns. D.Phil. thesis, University of Oxford, 2010. Forthcoming.

12. T. Murray and G. Lowe. On refinement-closed security properties and nondeterministic compositions. In Proceedings of AVoCS '08, pages 49-68, 2009.

13. G. T. Rohrmair and G. Lowe. Using data-independence in the analysis of intrusion detection systems. Theoretical Computer Science, 340(1):82-101, 2005.

14. A. W. Roscoe. The Theory and Practice of Concurrency. Prentice Hall, 1997.

15. A. W. Roscoe and P. J. Broadfoot. Proving security protocols with model checkers by data independence techniques. J. Comput. Secur., 7(2-3):147-190, 1999.

16. A. W. Roscoe and M. H. Goldsmith. What is intransitive noninterference? In Proceedings of CSFW'99, page 228. IEEE Computer Society, 1999.

17. P. Ryan and S. Schneider. Process algebra and non-interference. Journal of Computer Security, 9(1/2):75-103, 2001.

18. P. Y. A. Ryan. A CSP formulation of non-interference and unwinding. IEEE Cipher, pages 19-30, Winter 1991.

19. J. H. Saltzer and M. D. Schroeder. The protection of information in computer systems. Proceedings of the IEEE, 63(9):1208-1308, September 1975.

20. A. Spiessens. Patterns of Safe Collaboration. PhD thesis, Université catholique de Louvain, Louvain-la-Neuve, Belgium, February 2007.

21. R. van der Meyden. Architectural refinement and notions of intransitive noninterference. In Proceedings of ESSoS 2009, pages 60-74. Springer, 2009. 